\title{
LA SOSTENIBILIDAD COMO ESTRATEGIA COMPETITIVA EN EMPRESAS DEL SECTOR CONSTRUCCIÓN DEL DEPARTAMENTO DE ANTIOQUIA - COLOMBIA
}

\author{
Moisés Briñez* \\ htps://orcid.org/0000-0002-1126-017X \\ Manuela Penagos**
}

RECIBIDO: Julio 2020 / ACEPTADO: Febrero 2021 / PUBLICADO: Mayo 2021

Como citar: Briñez, Moises; Penagos, Manuela (2021). La Sostenibilidad como Estrategia Competitiva en empresas del sector Construcción del Departamento de Antioquia - Colombia. Telos: revista de Estudios Interdisciplinarios en Ciencias Sociales, 23 (2), Venezuela. (Pp.325-346).

DOI: www.doi.org/10.36390/telos232.08

\section{RESUMEN}

El desarrollo sostenible es un concepto bastante amplio que tiene implicaciones importantes en el ámbito corporativo. Las organizaciones deben enfrentarse no solo a retos relacionados con presiones de desempeño financiero, sino también a las crecientes expectativas de la sociedad para que actúen de forma más responsable en complejos contextos sociales, económicos y ambientales. A partir de lo anterior, el objetivo de este trabajo fue analizar la sostenibilidad como estrategia competitiva en las empresas del sector construcción en el departamento de Antioquia - Colombia. La investigación es de tipo descriptiva, con un enfoque cuantitativo, de modalidad exploratoria. Los principales autores que fundamentan teóricamente el estudio, están constituidos por: Bradford (2018), Bernyte (2018), Enshassi (2018), Wang et al., (2011), Weaver et al., (2008), Du Plessis (2007), Porter (2012), entre otros. Se diseñó un cuestionario con 51 ítems, el cual fue validado por el juicio de tres expertos; igualmente, para medir la confiabilidad se utilizó el coeficiente Alfa de Crombach, obteniendo un resultado de 0.94. La población estuvo conformada por todas las empresas registradas en la base de datos Galería Inmobiliaria, que para el año 2019 tenía registradas un total de 230 empresas. A través de un muestreo intencional, se seleccionaron 15 empresas, con el fin de poder entender bajo qué estrategias están ejecutando el cumpliendo de los estándares de sostenibilidad y poder así identificar en qué parámetros están mitigando los impactos que se encuentran dentro de los tres pilares de esta. Se determinó que las empresas del sector tienen una tendencia favorable en la implementación de la sostenibilidad en sus tres dimensiones en la gestión de sus

\footnotetext{
* Doctor en Ciencias Gerenciales. Profesor e investigador de la Universidad EIA. Correo electrónico: moises.brinez@eia.edu.co

** Ingeniera Administradora. Correo electrónico: manuela.penagos@eia.edu.co
} 
La sostenibilidad como estrategia competitiva en empresas del sector construcción del departamento de Antioquia - Colombia

proyectos y en las diferentes áreas, sin embargo, hoy se encuentra deficiencia en la implementación de éstas como ventaja competitiva ya sea por costos o diferenciación.

Palabras claves: gestión sostenible, estrategia competitiva, empresas de construcción, construcción, Colombia.

\section{Sustainability as a Competitive Strategy in companies in the Construction sector of the Department of Antioquia-Colombia}

\section{ABSTRACT}

Sustainable development is a fairly broad concept that has important implications at the corporate level. Organizations must face not only challenges related to financial performance pressures, but also the growing expectations of society to act more responsibly in complex social, economic and environmental contexts. Based on the above, the objective of this work was to analyze sustainability as a competitive strategy in companies in the construction sector in the department of Antioquia - Colombia. The research is descriptive, with a quantitative approach, exploratory in nature. The main authors who theoretically support the study are made up of: Bradford (2018), Bernyte (2018), Enshassi (2018), Wang et al., (2011), Weaver et al., (2008), Du Plessis (2007), Porter (2012), among others. A questionnaire with 51 items was designed, which was validated by the judgment of three experts; Likewise, to measure reliability, the Crombach Alpha coefficient was used, obtaining a result of 0.94 . The population made up of all registered companies was in the Real Estate Gallery database, which by 2019 had a total of 230 companies. Through an intentional sampling, 15 companies were selected, in order to understand under which strategies they are executing compliance with sustainability standards and thus be able to select in which parameters they are mitigating the impacts that are within the three pillars of this. It was determined that companies in the sector have a favorable trend in the implementation of sustainability in its three dimensions in the management of their projects and in the different areas, however, today there is a deficiency in the implementation of major as a competitive advantage, either by cost or differentiation.

Key words: sustainable management, competitive strategy, construction companies, construction, Colombia.

\section{Introducción}

En la actualidad se puede ver cómo las empresas se encuentran inmersas en entornos económicos, legales y políticos que buscan garantizar la sostenibilidad de los recursos, para el bienestar de la sociedad y de las futuras generaciones. Partiendo de la contribución al desarrollo económico de Colombia, la construcción es una de las actividades económicas que más aporta y contribuye el avance del mismo. Por tal motivo, las organizaciones empresariales requieren disponer de soluciones que permitan gestionar de manera sostenible los procesos medulares para mejorar los niveles de desempeño y competitividad. Es aquí donde se enfoca este sector, además de ser indispensable para el desarrollo de la sociedad, es también uno de los principales responsables de la generación de residuos, contaminación, transformación del entorno y uso considerable de energía. 
Tal como opina Martínez (2014), el crecimiento de la preocupación y la conciencia que están tomando las personas por las cuestiones medioambientales hace que las empresas promuevan comportamientos que tengan la aprobación y el apoyo de sus grupos de interés; es a causa de estos mismos que las prácticas de responsabilidad social aunado a la sostenibilidad, empiezan a jugar un papel fundamental dentro de la estrategia empresarial como consecuencia de la globalización de los mercados así como las exigencias y demandas de una mayor transparencia con la sociedad.

El sector construcción utiliza una gran cantidad de fuentes de energía usando recursos renovables y es por esto que debido a las condiciones críticas de daño ecológico e impacto climático que está teniendo el planeta el tema de las construcciones sostenibles ha tenido mucha más acogida dentro de las empresas. Prueba de ello, es que en el país cada vez son más frecuentes los proyectos de alto desempeño y sostenibilidad integral para mitigar el cambio climático y garantizar el bienestar de las comunidades y el cuidado del medio ambiente (Argos, 2018).

Partiendo de lo anterior, queda claro que la sostenibilidad debe ser concebida entonces como estrategia de negocio, ya que las personas, el entorno y el medio ambiente son quienes soportan las actividades que realiza una organización. Por lo tanto, transformarse en una compañía sostenible es una forma de invertir en el futuro de las siguientes generaciones y un medio de permanencia en el mercado por más tiempo y con una mayor rentabilidad (Bradford, 2018).

El objetivo de esta investigación estuvo orientado en analizar la sostenibilidad como estrategia competitiva por las empresas del sector construcción. Se utilizaron fuentes de información de tipo primario y secundario. Los resultados permitieron evidenciar bajo qué estrategias están ejecutando el cumpliendo de la sostenibilidad y poder así identificar en que parámetros están mitigando los impactos que se encuentran dentro de los tres pilares de esta.

\section{Sostenibilidad y estrategias competitivas en el sector construcción.}

Actualmente, el mundo se encuentra en una época de enormes transformaciones demográficas, tecnológicas y económicas. En un intento por asegurar que los cambios que afectan a la humanidad sean para mejor, la comunidad mundial ha iniciado el proceso de redefinición del progreso. Este intento de redefinir el progreso, es lo que se conoce como desarrollo sostenible (Gallopín, 2003). Bajo este mismo contexto, el autor indica que la velocidad y magnitud del cambio global cada vez mayor de las sociedades y de sus impactos sobre la biosfera, ponen de relieve que el desarrollo sostenible debe orientarse no tanto a preservar como mantener la base ecológica del desarrollo, también a aumentar la capacidad social - ecológica de hacer frente al cambio, la capacidad de conservar y ampliar las opciones disponibles para confrontar un mundo natural en permanente transformación.

Lo expuesto anteriormente, pretende crear conciencia e iniciativa para generar un cambio positivo y un desarrollo de impacto personal que se visualiza mejor en conjunto. Por lo tanto, aplicarlo a un nivel empresarial ya no se trata solo de si es necesario o legal, es algo indispensable hoy en día en una empresa u organización. Se trata de una filosofía que permite generar dicho cambio positivo (Rubio, 2012). Tal como indica Heincke (2005), es un proceso de concientización, una ética, una visión y hasta una forma de ver el mundo que en Colombia se está construyendo. 
La sostenibilidad como estrategia competitiva en empresas del sector construcción del departamento de Antioquia - Colombia

En este sentido, de acuerdo a Rodríguez (2012), una empresa sostenible es aquella que crea valor económico, medioambiental y social a corto y largo plazo, contribuyendo de esa forma al aumento del bienestar y al auténtico progreso de las generaciones presentes y futuras, en su entorno general. La sostenibilidad trabaja con base a tres pilares, que implican las áreas: económicas, ambiental y social. La primera implica generar riqueza económica en un marco local, regional y global que estimule el desarrollo financieramente posible y rentable, manteniendo la base de los recursos naturales y su conservación. La ambiental, se fundamenta en lograr la compatibilidad entre las actividades humanas y la preservación de la biodiversidad y de los ecosistemas; y tercero, la social, se basa en el mantenimiento de la red social y cultural, de la capacidad para mantener intereses comunes por vías democráticas y no excluyentes.

Bajo este orden de ideas, LatinAmerican Post (2019), explica que las empresas deben definir la forma en que administrarán los recursos (ambientales y sociales) es decir, las entidades empresariales no pueden únicamente dedicarse a producir riqueza y generar empleo, deben potenciar y ayudar al florecimiento de la sociedad.

En este sentido, es necesario conocer los métodos y técnicas se están implementando en el sector para identificar la aplicabilidad de la sostenibilidad en las empresas del sector construcción del departamento de Antioquia. Para lograr esto, se consultaron diferentes investigaciones desde lo local e internacional, para disponer de un marco de referencia global que asegure una medición objetiva en el sector construcción; tales como: Acevedo et al. (2012), Enshassi (2018), Álvaro (2016), Fernández (2014); Wang et al., (2011), Li-Yin et al., (2011), Weaver et al., (2008), Du Plessis (2007) y, Hoof y Herrera (2007).

No obstante, se destacan los dos indicadores que sirvieron de base para la discusión de este artículo. En primer lugar, la arquitectura y diseño sostenible es un término que describe las técnicas de diseño ambientalmente adecuadas en el campo de la arquitectura, buscando minimizar el impacto ambiental negativo de los edificios al incrementar su eficiencia y moderación en el uso de materiales y energía y el desarrollo del espacio.

Ahora bien, es importante tener presente lo indicado por Li-Yin et al., (2011), ya que señalan que no deben existir esfuerzos aislados o únicamente en una fase del proyecto; es necesario emplear metodologías integradoras que aseguren un trabajo coherente y cooperativo desde la fase de diseño, así como durante toda la ejecución. Bajo ese orden de ideas, Hoof y Herrera (2007), destacan que existe la necesidad de un conjunto de instrumentos integradores y mecanismos para crear valor agregado a la gestión sostenible de las empresas. Para Villa (2009) la práctica sostenible tiende a promover la eficiencia en el manejo de la energía y el uso de productos que son más seguros, renovables y reciclables, y reducen desperdicios dañinos en su producción. Un diseño sostenible exitoso es el resultado de un proceso integrado en la toma de decisiones, empezando desde el comienzo del proyecto.

En relación a esto, Acevedo et al. (2012), indican que es propio mencionar la arquitectura bioclimática, siendo una herramienta cuyo propósito es concebir proyectos que mantengan el uso eficiente de los recursos, aprovechando las consideraciones del entorno con la finalidad de disminuir el consumo energético Se puede encontrar casos, dignos de mención, de diseños concebidos bajo parámetros bioclimáticos. En Colombia, proyectos como el Orquideorama y la adecuación de la Biblioteca Pública Piloto en Medellín, la urbanización La Aldea en La Estrella, la ciudadela ecológica Nashira en el Valle del Cauca, el Colegio San José 
y el proyecto de recuperación del centro histórico de Barranquilla y el Pueblito Acuarela en Santander son exponentes de la aplicación de conceptos bioclimáticos.

En este sentido, Enshassi (2018), opina que en el alcance del diseño sostenible se debe incluir la regulación ambiental y promoción del uso eficiente de los proyectos de infraestructura. La experiencia de las evaluaciones medioambientales demuestra que los proyectos de construcción tienen menos probabilidades de causar estrés en el medio ambiente si esas evaluaciones se realizan con antelación e influyen en el diseño de los proyectos. Con respecto al impacto ambiental, Weaver et al., (2008), hacen énfasis en que la evaluación del impacto ambiental, sigue siendo la principal herramienta reguladora utilizada en todo el mundo, en materia de desarrollo sostenible. Por su parte, Fernández (2014), destaca que el Departamento Nacional de Planeación (DNP) establece códigos de construcción sostenibles enfocados en garantizar edificaciones que incorporen elementos clave como la gestión de calidad de vida, uso de materiales ecológicos, uso eficiente y racional de energía; la conservación, ahorro y reutilización del agua; utilización de recursos reciclables y renovables, reducción de residuos, emisión de gases, entre otros.

Bajo el mismo orden de ideas, la ecoeficiencia se obtiene por medio del suministro de bienes y servicios con precios competitivos, que satisfacen las necesidades humanas y dan calidad de vida, al tiempo que reducen progresivamente los impactos ecológicos y la intensidad de uso de los recursos a lo largo de su ciclo de vida, a un nivel por lo menos acorde con la capacidad de carga estimada de la tierra. Ser más eficiente tiene sentido empresarial y social, ya que ayuda a las organizaciones a que obtengan más valor, con un menor consumo de materiales y energía y con una reducción en las emisiones (Ministerio del Ambiente Perú, 2009).

De acuerdo a Leal (2005), la ecoeficiencia apunta claramente no sólo en esa dirección sino también en el tratamiento de los recursos naturales, tanto materias primas como insumos energéticos. Es un enfoque que se interna en la operación las empresas mismas y no se queda en las externalidades (emisiones, efluentes, residuos), forma tradicional de tratar el tema.

En este sentido, Du Plessis (2007), señala que para que se pueda emprender un camino de desarrollo y construcción sostenible, se requiere un enfoque de dos vertientes: primero es necesario crear un sector de la construcción local capaz y viable; segundo, es necesario asegurar que el sector sea capaz de responder a las demandas que el desarrollo sostenible impone a sus actividades. Lo cual solo puede ser posible si todas las partes interesadas cooperan en la implementación de una estrategia clara que implique acciones de apoyo específicas por parte de todos los actores y el desarrollo de un conjunto de facilitadores.

Ahora bien, queda claro que la sostenibilidad debe ser concebida entonces como estrategia de negocio, ya que las personas, el entorno y el medio ambiente son quienes soportan las actividades que realiza una organización. Por lo tanto, transformarse en una compañía sostenible es una forma de invertir en el futuro de las siguientes generaciones y un medio de permanencia en el mercado por más tiempo y con una mayor rentabilidad (Bradford, 2018).

Es por esto por lo que las empresas han enfocado sus estrategias no solo en lo que demandan los consumidores, sino que se han encargado de transmitir los valores y principios que rigen a la compañía; esa es la razón por la cual los usuarios están dispuestos a comprar 
La sostenibilidad como estrategia competitiva en empresas del sector construcción del departamento de Antioquia - Colombia

más de las empresas que comparten sus ideales, y mayormente si estos están orientando hacia el desarrollo sostenible (Bernyte, 2018).

De manera particular, en Colombia el desarrollo sostenible es un tema obligatorio dentro del cumplimiento de las organizaciones y donde sus estrategias se deben ver reflejadas en el ámbito social, ambiental y económico. Por tanto, en la defensa de la sostenibilidad ambiental de los grandes proyectos nacionales, han salido al ruedo los organismos gubernamentales de vigilancia y control como la Procuraduría General de la Nación y la Contraloría General de la República. Por otra parte, muchas industrias han tenido que reestructurarse y otras retirarse, pero así mismo han surgido también nuevas industrias mucho más limpias y eficientes con el uso de los recursos (Galvis, 2009).

Siguiendo con el orden ideas, las empresas por naturaleza son consumistas y competitivas, lo cual conlleva a que sus gestiones se construyan con innumerables situaciones que, en medio de la cotidianidad, las hacen competir en el cumplimiento de sus objetivos y permanecen en una continua exploración de formas que mejoren sus condiciones. Sin embargo, todas las empresas por su mismo carácter consumista, si no acompañan su gestión empresarial con términos de sostenibilidad, significan una amenaza no solo para la sociedad y el medio ambiente, sino para sí mismas, pues carecen de sentido propio y reputación en el mercado.

De igual forma, las empresas se obligan a estar en una búsqueda constante de estrategias que les permitan el logro de sus objetivos. Es allí donde, según Meza (2007), existe la urgente necesidad que todas las empresas adopten una gestión sostenible, obligando a éstas a diseñar mecanismos a fin de obtener el valor estratégico de ello y consolidar sus ventajas competitivas. A este respecto, Álvaro (2016), indica que cada día en mayor medida, las empresas se han visto motivadas a situar las prácticas responsables y los objetivos de sostenibilidad en el centro de sus estrategias.

Dentro del conjunto genérico de las estrategias de negocio se encuentran las estrategias competitivas, que tienen como objetivo que la empresa pueda mejorar su posición con respecto a las empresas del sector. Esto se puede lograr de diferentes maneras, pero una estrategia de este tipo, tiene como objetivo fundamental la obtención de una ventaja competitiva, es decir, la definición de una característica o de una cualidad que haga que la empresa supere a la competencia de manera genérica y sostenida (BBVA, 2019).

Según Porter (2012), el concepto de las estrategias se basa en que la ventaja competitiva constituye la esencia de cualquier estrategia y en que para lograrla es indispensable tomar una decisión; si la compañía quiere alcanzar la ventaja competitiva, deberá escoger la clase que desea obtener y el ámbito donde lo hará: liderazgo por costos, diferenciación y enfoque. Vale destacar que, para efectos de esta investigación, la medición práctica en el sector construcción, se enfocará en el liderazgo por costos y diferenciación; en vista que el modelo de negocio de las empresas objeto de estudio no persigue una estrategia de enfoque.

Siguiendo con el orden de ideas, como opina Cruz y Álvarez (2014), la ventaja competitiva debe ser única con un diferenciador principal que lo posicione en el mercado como líder. Es algo que solo él posee y los demás competidores no. Es la que realmente le dará la ventaja sobre los competidores, pero también es primordial que las empresas no se queden solo con una eso, ya que los competidores también van evolucionando y tarde o temprano 
ellos también contaran con sus ventajas. Por lo que se debe estar siempre en continuo análisis de su ambiente interno y externo, modificando y modernizando esas ventajas competitivas.

En relación a ello, el Consejo Colombiano de Construcción Sostenible (CCCS), tiene como propósito asegurar que se realicen mejores prácticas de urbanismo y construcción sostenible, entornos prósperos y ambientalmente responsables, inclusivos y saludables para todos. Hoy el CCCS tiene como aliado estratégico al Green Business Certificación INC. (GBCl) para el programa LEED en Colombia, donde es reconocida mundialmente como una certificación de excelencia en construcciones sostenibles. En el 2018, se encontraban 151 proyectos con certificación LEED y 223 en proceso, lo que indica una gran responsabilidad en el sector de concretar oportunidades para la evolución de la industria de la construcción hacia la sostenibilidad integral (CCCS, 2018).

Tal como indica Fernández (2014), se trata de actuar bajo una construcción sostenible, la cual tiene que ver con la mejoría de las prácticas durante el ciclo de vida de las edificaciones, es decir desde el diseño, ejecución y operación de los mismos, los cuales aportan de forma efectiva a minimizar el impacto que este sector puede tener en el cambio climático por sus emisiones de gas carbónico, el consumo de los recursos naturales y la pérdida de la biodiversidad. Como señala Garzón e Ibarra (2014), lo anterior se garantiza cuando la empresa introduce prácticas de actuación respetuosas con el medio ambiente como un continuo que va desde programas básicos hasta iniciativas complejas y exigentes de naturaleza estratégica.

Es importante destacar que Rubio (2012), opina que el desarrollo empresarial en las organizaciones del sector constructor en Colombia tienen mucho potencial para ser competitivas en un mercado, pero a su vez estas tienen la necesidad de evolucionar en su plano estructural dentro de un proceso de transformación cultural que permita tener una visión integral sostenible de la gestión corporativa e involucre mejores prácticas, desarrollo continuo del capital humano, planeación estratégica flexible para estar preparadas y hacer lo necesario en el momento de responder a los cambios rápidos del entorno, empresas interconectadas con los actores del sector, y conducirlas a la generación de sociedades de conocimiento con capital intelectual que las haga diferentes a las demás. Además de esto, según Villa (2009), el diseño y las construcciones sostenibles pueden proveer beneficios económicos, ambientales y sociales que son el resultado del cuidado al considerar el uso de los recursos.

En síntesis, la investigación buscó medir el comportamiento de las variables: sostenibilidad, con las tres dimensiones: social, económica y ambiental; su aplicación, desde la arquitectura sostenible y ecoeficiencia y; las estrategias competitivas en liderazgo por costos, así como por diferenciación.

\section{Metodología.}

En relación con el objetivo del presente estudio y el tipo de problema presentado se realizó una investigación exploratoria descriptiva, pues se buscó especificar y recoger información sobre la sostenibilidad como estrategia competitiva en las empresas del sector construcción.

Se investigaron diferentes definiciones teóricas, obteniendo una sólida base a fin de dar consistencia a los resultados de esta. Vale destacar que, en virtud de encontrarnos inmersos en el globalizado mundo empresarial, las investigaciones utilizadas como soporte 
La sostenibilidad como estrategia competitiva en empresas del sector construcción del departamento de Antioquia - Colombia

teórico están dentro de una margen de 5 años de antigüedad, evitando que la información seleccionada careciera de pertinencia y vigencia, con relación a la realidad del contexto abordado. Las palabras utilizadas para las consultas en las bases de datos Scopus, Sciencie Direct, Scielo, Google Scholar, Redalyc, empleando el idioma español e inglés, fueron: Sostenibilidad (sustainability), estrategias competitivas (Competitive strategies) y empresas del sector construcción (Companies in the construction sector).

Se aplicó un cuestionario autoadministrado, puesto que se proporcionaron las preguntas en un formulario escrito con las instrucciones incluidas. Los encuestados respondieron solos sin la intervención del investigador. En el instrumento se utilizó una escala de medición Likert, donde las opciones de respuesta son fijas para todos los ítems y todos tienen designado un peso o valor similar o equivalente. La encuesta se dividió en dos (2) variables, las cuales eran la gestión sostenible y las estrategias competitivas.

Vale destacar que, el contenido del instrumento se validó a través de la revisión de tres (3) expertos en temas referidos a las variables objeto de estudio, así como demostrado dominio en metodología de la investigación. Con base a la revisión, se realizaron las correcciones pertinentes a los ítems que presentaron dificultad, ambigüedad o no correspondencia con el estudio, tomándose éstas en cuenta para mejorar el cuestionario y diseñando el instrumento final validado por los expertos. La confiabilidad del instrumento se determinó utilizando el método de coeficiente Alfa Crombach y el resultado obtenido fue de 0,94., considerando que es un buen valor para afirmar que se tiene un instrumento confiable.

La población estuvo conformada por todas las empresas registradas en la base de datos Galería Inmobiliaria, que para el año 2019 tenía registradas un total de 230 empresas, razón que obedece a que institucionalmente agrupa y representa a las empresas y entidades de la cadena productiva de la construcción y contribuye significativamente a la consolidación de la actividad edificadora, a la dinamización del sector y al desarrollo de Antioquia.

En este sentido, se buscó conocer cuáles de estas empresas se encuentran operando en Antioquia y así mismo entender bajo qué estrategias están ejecutando el cumpliendo de los estándares de sostenibilidad. Esto con la finalidad de conocer el compromiso que tienen las organizaciones que se ubican dentro del sector y así identificar en que parámetros están mitigando los impactos que se encuentran dentro de los tres pilares de la sostenibilidad. Se utilizó un muestreo intencional, quedando determinada por 15 empresas seleccionadas a partir de los siguientes criterios: primero, el acceso para la recolección de datos; segundo, que a la fecha tengan alguna certificación sostenible o que estén en vía de la obtención y tercero, la ejecución continua de proyectos en el departamento de Antioquia.

\section{Resultados.}

La aplicación de la sostenibilidad empresarial en el sector se consideró evaluando la sostenibilidad, sus tres pilares social, ambiental y económica; además de la aplicación como estrategias competitivas. En primer lugar, se evaluó la sostenibilidad en términos generales identificando que tan presente tenía la empresa los principios del pacto global y su incidencia en cada una de sus áreas. Los resultados se muestran en la tabla №1. 
Tabla 1. Tendencias indicador sostenibilidad

\begin{tabular}{|c|c|c|c|c|}
\hline $\begin{array}{l}\text { Indicador: } \\
\text { Sostenibilidad }\end{array}$ & $\begin{array}{l}\text { La filosofía de } \\
\text { gestión de la } \\
\text { empresa tiene } \\
\text { coherencia con } \\
\text { la postura de } \\
\text { esta frente a la } \\
\text { sostenibilidad }\end{array}$ & $\begin{array}{l}\text { Los directores } \\
\text { de cada área } \\
\text { analizan los } \\
\text { impactos de su } \\
\text { operación a } \\
\text { nivel social }\end{array}$ & $\begin{array}{l}\text { Los directores } \\
\text { de cada área } \\
\text { analizan los } \\
\text { impactos de su } \\
\text { operación a } \\
\text { nivel ambiental }\end{array}$ & $\begin{array}{lr}\text { Los directores } \\
\text { de cada área } \\
\text { analizan los } \\
\text { impactos de su } \\
\text { operación a } \\
\text { nivel } \\
\text { económico } \\
\end{array}$ \\
\hline $\begin{array}{l}\text { Tendencia Positiva (TA+ } \\
\text { DA) }\end{array}$ & 73, & 66 & $\%$ & $93,3 \%$ \\
\hline Indiferencia (NN) & $13,3 \%$ & $20,0 \%$ & $0,0 \%$ & $6,7 \%$ \\
\hline $\begin{array}{l}\text { Tendencia Negativa (ED+ } \\
\text { TD) }\end{array}$ & $13,3 \%$ & $13,3 \%$ & $13,3 \%$ & $0,0 \%$ \\
\hline TOTAL & $100 \%$ & $100 \%$ & $100 \%$ & $100 \%$ \\
\hline
\end{tabular}

Fuente: elaboración propia.

El 73,3\% afirma que la filosofía de gestión tiene coherencia con la postura de esta frente a la sostenibilidad y se relaciona con el análisis del impacto que realizan los directores de cada área en su operación a nivel de los 3 pilares de la sostenibilidad. En efecto, la mayor parte de las empresas están de acuerdo frente al análisis del impacto que tienen los directores de cada área de su operación en un $66,7 \%$ a nivel social, el $86,7 \%$ a nivel ambiental y el mayor porcentaje de un $93,3 \%$ frente al impacto a nivel económico.

En términos generales, este indicador tuvo una tendencia positiva evidenciando que el sector construcción está tomando postura frente a la implementación de estrategias que se alinean con la sostenibilidad corporativa en aspectos ambientales, sociales y económicos. Esto ayuda en la movilización de los esfuerzos que se están haciendo a nivel global alrededor de los objetivos y metas pactadas, con el fin de minimizar los impactos negativos y maximizar los positivos en pro de las personas y el cuidado del planeta.

Seguidamente, tal como se evidencia en la tabla №2 evaluando el indicador del primer pilar de la sostenibilidad que es el social, según el primer ítem el $100 \%$ de las empresas afirman que tienen parámetros y normativas para generar un entorno seguro y saludable para trabajar. Además, el $80 \%$ de las organizaciones tienen una tendencia positiva ya que indican que están de acuerdo en que la compañía permite que los colaboradores puedan equilibrar en su vida la parte laboral, de salud y familiar, mientras que un 13,3\% está en desacuerdo. También, los encuestados reconocen en un $80 \%$ que la compañía tiene una postura equitativa en igualdad de salarios sin importar el género, el $13,3 \%$ no tiene conocimiento de esto y el $6,7 \%$ están en desacuerdo.

Con relación a la posición que tienen las empresas frente al apoyo en la ejecución de las actividades sostenibles, el $100 \%$ de los encuestados están de acuerdo en que sus organizaciones lo realizan. Un aspecto fundamental para las empresas es poder analizar las posturas que tienen los proveedores, los socios y los subcontratistas frente a la sostenibilidad empresarial, con el fin de trabajar en conjunto bajo aquellos lineamientos que mitiguen los impactos a nivel de la operación. El 53,3\% de las empresas afirman que dentro de su organización se realizan estos análisis, sin embargo, el $26,7 \%$ de las encuestados de las 
La sostenibilidad como estrategia competitiva en empresas del sector construcción del departamento de Antioquia - Colombia

empresas no tiene conocimiento de que esto se realice con los proveedores, el $33,3 \%$ de que se realice con los socios y el $20 \%$ con los subcontratistas.

Tabla 2. Tendencias Indicador sostenibilidad social

\begin{tabular}{|c|c|c|c|c|c|c|c|}
\hline $\begin{array}{l}\text { Indicador: } \\
\text { Social }\end{array}$ & $\begin{array}{l}\text { La } \\
\text { empresa } \\
\text { tiene } \\
\text { parámetr } \\
\text { os y y } \\
\text { normas } \\
\text { para } \\
\text { generar } \\
\text { un } \\
\text { entorno } \\
\text { seguro y } \\
\text { saludable } \\
\text { para } \\
\text { trabajar }\end{array}$ & $\begin{array}{l}\text { Se realiza } \\
\text { un análisis } \\
\text { de } \\
\text { proveedore } \\
\text { s para } \\
\text { conocer su } \\
\text { postura } \\
\text { frente a la } \\
\text { sostenibilid } \\
\text { ad } \\
\text { empresarial }\end{array}$ & $\begin{array}{l}\text { Se realiza } \\
\text { un análisis } \\
\text { de socios } \\
\text { para } \\
\text { conocer su } \\
\text { postura } \\
\text { frente a la } \\
\text { sostenibilid } \\
\text { ad } \\
\text { empresarial }\end{array}$ & $\begin{array}{l}\text { Se realiza un } \\
\text { análisis de } \\
\text { subcontratist } \\
\text { as para } \\
\text { conocer su } \\
\text { postura } \\
\text { frente a la } \\
\text { sostenibilida } \\
\text { d } \\
\text { empresarial }\end{array}$ & $\begin{array}{l}\text { Las } \\
\text { actividades } \\
\text { de trabajo } \\
\text { permiten } \\
\text { que los } \\
\text { colaborador } \\
\text { es puedan } \\
\text { equilibrar en } \\
\text { su vida la } \\
\text { parte } \\
\text { laboral, de } \\
\text { salud y } \\
\text { familiar }\end{array}$ & $\begin{array}{l}\text { La } \\
\text { compañí } \\
\text { a tiene } \\
\text { una } \\
\text { postura } \\
\text { equitativ } \\
\text { a en } \\
\text { igualdad } \\
\text { de } \\
\text { salarios } \\
\text { sin } \\
\text { importar } \\
\text { el } \\
\text { género }\end{array}$ & $\begin{array}{l}\text { Se } \\
\text { realiza un } \\
\text { diagnósti } \\
\text { co para el } \\
\text { público al } \\
\text { cual se } \\
\text { dirige la } \\
\text { obra, a } \\
\text { objeto de } \\
\text { identificar } \\
\text { beneficio } \\
\text { s directos }\end{array}$ \\
\hline $\begin{array}{l}\text { Tendencia } \\
\text { Positiva (TA+ } \\
\text { DA) }\end{array}$ & $100,0 \%$ & $53,3 \%$ & $53,3 \%$ & $53,3 \%$ & $80,0 \%$ & $80,0 \%$ & $66,7 \%$ \\
\hline $\begin{array}{l}\text { Indiferencia } \\
\text { (NN) }\end{array}$ & $0,0 \%$ & $26,7 \%$ & $33,3 \%$ & $20,0 \%$ & $6,7 \%$ & $13,3 \%$ & $20,0 \%$ \\
\hline $\begin{array}{l}\text { Tendencia } \\
\text { Negativa (ED+ } \\
\text { TD) }\end{array}$ & $0,0 \%$ & $20,0 \%$ & $13,3 \%$ & $26,7 \%$ & $13,3 \%$ & $6,7 \%$ & $13,3 \%$ \\
\hline TOTAL & $100 \%$ & $100 \%$ & $100 \%$ & $100 \%$ & $100 \%$ & $100 \%$ & $100 \%$ \\
\hline
\end{tabular}

Fuente: elaboración propia

De acuerdo a los resultados que se obtuvieron en la tabla de tendencias del indicador social, se puede evidenciar que las empresas en el sector tienen una mayor conciencia social en todos los ámbitos, desde el trato y contrataciones con los empleados, acuerdos con proveedores y hasta la planeación de estrategias y beneficios para las personas que gozarán de los proyectos que ejecuta cada organización. Incorporar la sostenibilidad dentro de las empresas, requiere un gran compromiso de las personas que dirigen dentro de ellas, impulsando ciertos valores y logrando que toda la organización trabaje en pro de estos. Esto corresponde con la investigación efectuada por Enshassi (2018), quien demostró que las empresas constructoras consideran primordial la conciencia y comunicación ciudadana con todos sus Stakeholders, desde el inicio hasta la culminación de cada obra.

En la tabla $\mathrm{N}^{\circ} 03$ se puede observar que el $86,7 \%$ de los encuestados confirman que las empresas del sector construcción diseñan planes estratégicos que promueven una rentabilidad y estabilidad a largo plazo. Sin embargo, solo el 33,3\% de las empresas se someten a mediciones para participar en índices de inversión responsable y el $60 \%$ confirman que no tienen conocimiento de que esto se haga. Por otro lado, con una tendencia positiva del $93,3 \%$ se confirma que las empresas emplean de manera eficiente los recursos y materiales 
que se tienen destinados para cada proyecto, lo que implica que están usando menos y de mejor manera lo recursos incluyendo las materias primas, reduciendo la utilización de materiales no renovables e incrementando la utilización de los renovables.

Tabla 3. Tendencias Indicador sostenibilidad económica

\begin{tabular}{|c|c|c|c|c|c|}
\hline $\begin{array}{l}\text { Indicador: } \\
\text { Económica }\end{array}$ & $\begin{array}{l}\text { La empresa se } \\
\text { somete a } \\
\text { mediciones } \\
\text { para participar } \\
\text { en índices de } \\
\text { inversión } \\
\text { responsable }\end{array}$ & $\begin{array}{l}\text { La compañía } \\
\text { diseña planes } \\
\text { estratégicos } \\
\text { que promueven } \\
\text { una rentabilidad } \\
\text { y estabilidad a } \\
\text { largo plazo }\end{array}$ & $\begin{array}{l}\text { Se emplean de } \\
\text { manera } \\
\text { eficiente los } \\
\text { recursos y } \\
\text { materiales que } \\
\text { se tienen } \\
\text { destinados } \\
\text { para cada } \\
\text { proyecto }\end{array}$ & $\begin{array}{l}\text { Se administran de } \\
\text { manera efectiva } \\
\text { las actividades } \\
\text { que se deben } \\
\text { ejecutar, } \\
\text { programando el } \\
\text { tiempo requerido } \\
\text { para cada una de } \\
\text { éstas }\end{array}$ & $\begin{array}{l}\text { Se evalúa la } \\
\text { modalidad de } \\
\text { contrato que } \\
\text { garantice } \\
\text { mayor } \\
\text { rendimiento } \\
\text { con todos los } \\
\text { contratistas }\end{array}$ \\
\hline $\begin{array}{l}\text { Tendencia } \\
\text { Positiva (TA+ DA) }\end{array}$ & $33,3 \%$ & $86,7 \%$ & $93,3 \%$ & $80,0 \%$ & $73,3 \%$ \\
\hline Indiferencia (NN) & $60,0 \%$ & $6,7 \%$ & $0,0 \%$ & $6,7 \%$ & $13,3 \%$ \\
\hline $\begin{array}{l}\text { Tendencia } \\
\text { Negativa (ED+ } \\
\text { TD) }\end{array}$ & $6,7 \%$ & $6,7 \%$ & $6,7 \%$ & $13,3 \%$ & $13,3 \%$ \\
\hline TOTAL & $100 \%$ & $100 \%$ & $100 \%$ & $100 \%$ & $100 \%$ \\
\hline
\end{tabular}

Fuente: elaboración propia

Otra de las medidas utilizadas dentro de las empresas, es administrar de manera efectiva las actividades que se deben ejecutar, el $80 \%$ están de acuerdo con la programación del tiempo que requieren cada una de éstas y el $13,3 \%$ expresan no estarlo. Cabe resaltar que una planificación inteligente es la clave del éxito en la gestión de proyectos. Con base en lo anterior, una de las mejores prácticas a la hora de establecer acuerdos comerciales con contratistas para una buena gestión del proyecto es evaluar una modalidad de contrato que garantice mayor rendimiento, el $73,3 \%$ de las empresas confirman que lo realizan, lo que permite el desarrollo de los proyectos sin mayores contratiempos. Dichos resultados son congruentes con la investigación de Hill y Bowen (2010), quienes infieren que una gestión efectiva desde el inicio minimiza el costo total y asegura la asequibilidad financiera del proyecto.

En Antioquia, es evidente que las empresas del sector están generando un crecimiento económico de la mano de planeaciones estratégicas y administración eficiente de recursos, que hacen posible la creación de valor de crecimiento en el corto y el largo plazo. En general las empresas encuestadas tienen una tendencia positiva, sin embargo, se debe seguir trabajando en la construcción de los índices de inversión responsable, teniendo en cuenta los 
La sostenibilidad como estrategia competitiva en empresas del sector construcción del departamento de Antioquia - Colombia

impactos sociales y ambientales derivados, con el fin de buscar una rentabilidad financiera competitiva y además reconocimientos internacionales dentro de las empresas más sostenibles del sector.

En la tabla Nº se evalúa el indicador de medio ambiente que es el tercer pilar de la sostenibilidad empresarial, el $73,3 \%$ de los encuestados afirman que para la compañía es fundamental mitigar los efectos ambientales que se generan en los procesos de construcción y el $20 \%$ están en desacuerdo. Esto muestra que las empresas de construcción si están mostrando interés en tomar acciones concretas que demuestren el compromiso en la conservación del medio ambiente.

Tabla 4. Tendencias Indicador sostenibilidad ambiental.

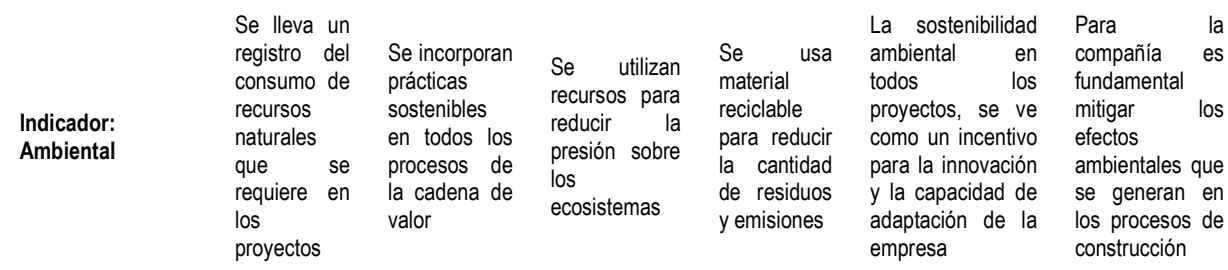

\begin{tabular}{|c|c|c|c|c|c|c|}
\hline $\begin{array}{l}\text { Tendencia } \\
\text { Positiva } \\
\text { DA) }\end{array}$ & $40,0 \%$ & $73,3 \%$ & $66,7 \%$ & $73,3 \%$ & $53,3 \%$ & $73,3 \%$ \\
\hline Indiferencia (NN) & $33,3 \%$ & $6,7 \%$ & $13,3 \%$ & $13,3 \%$ & $20,0 \%$ & $6,7 \%$ \\
\hline $\begin{array}{l}\text { Tendencia } \\
\text { Negativa (ED+ } \\
\text { TD) }\end{array}$ & $26,7 \%$ & $20,0 \%$ & $20,0 \%$ & $13,3 \%$ & $26,7 \%$ & $20,0 \%$ \\
\hline TOTAL & $100 \%$ & $100 \%$ & $100 \%$ & $100 \%$ & $100 \%$ & $100 \%$ \\
\hline
\end{tabular}

Fuente: elaboración propia

Con relación a esto, el $73,3 \%$ de las empresas incorporan prácticas sostenibles en todos los procesos de la cadena de valor, lo que indica que se tiene en cuenta desde el proceso de fabricación de los materiales, la fase de construcción, utilización y vida útil del proyecto. Del mismo modo, Li-Yin et al., (2011), revelaron que cada día con mayor auge, los efectos medioambientales son ampliamente tratados, entre ellos: el consumo energético, emisión de polvo y gases, generación de residuos, descargas de aguas, mal uso de recursos hídricos, entre otros.

De acuerdo a la tabla $\mathrm{N}^{\circ} 04$, el $40 \%$ confirma que la organización lleva un registro del consumo de recursos naturales que se requiere en los proyectos y el $60 \%$ de los integrantes de la organización no están de acuerdo con que esto se realice 0 no tienen conocimiento, lo que es indispensable en la sostenibilidad de las organizaciones en el largo 
plazo, y más aún si la empresa tiene actividades que se relacionan con la explotación y extracción de los recursos naturales.

Sin embargo, cabe resaltar que un $73,3 \%$ respondió que estaba de acuerdo en que la compañía usa material reciclable para reducir la cantidad de residuos y emisiones y en un $66,7 \%$ de que se utilizan recursos para reducir la presión sobre los ecosistemas; es importante considerar que estas estrategias dentro de las compañías que se encuentran dentro del sector construcción se deben tener en cuenta desde el diseño y en las demás etapas del proyecto. Es por esto que es importante que las empresas promuevan este tipo de políticas, ya que de un $100 \%$ apenas un $53,3 \%$ de los encuestados afirman que la sostenibilidad ambiental en todos los proyectos se ve como un incentivo para la innovación y la capacidad de adaptación de la empresa.

Tal como lo muestra uno de los referentes científicos de esta investigación (Acevedo et al., 2012), donde diagnosticaba la situación del sector construcción en relación al impacto ambiental que este genera, existe una correspondencia con el tipo de los resultados ya que se nota un gran desconocimiento por el personal encargado de las diferentes áreas con lo referente a la sostenibilidad en las empresas del sector. No obstante, muchas de ellas si están realizando una buena implementación dentro de sus prácticas y medidas ambientales, generando un crecimiento económico y una buena administración eficiente de los recursos con una tendencia de crecimiento en el corto y el largo plazo. Esto indicia que, a diferencia de la investigación antes citada, las implementaciones de estas prácticas no las están viendo como una afectación directa a los costos de manera negativa y que por el contrario sí generan beneficios económicos.

Por otro lado, para analizar la aplicación de la sostenibilidad se tuvieron en cuenta dos indicadores claves que fueron la arquitectura y diseño sostenible y la ecoeficiencia. El sector de construcción utiliza una gran cantidad de fuentes de energía usando recursos renovables lo que implica un gran impacto para el medio ambiente, lo que representa un papel muy importante en el desarrollo de estrategias de la organización para minimizar esos impactos negativos que se generen por causa de esto. Es por esto por lo que se hace énfasis en la importancia que tiene la aplicación de estas medidas en todas las etapas por las que pasa un proyecto de construcción.

La arquitectura y diseño sostenible implica la utilización de técnicas y el uso de materiales respetuosos con el medio ambiente para el desarrollo de un proyecto de construcción, donde se tiene en cuenta las condiciones del sitio buscando minimizar los impactos negativos a través de un consumo eficaz de los recursos naturales.

Como se puede observar en la tabla $\mathrm{N}^{\circ} 05$, la etapa de planeación y diseño es una de las más importantes para regular todo el acondicionamiento de trabajo y evaluar todas las posibilidades ante los desafíos que se enfrentan en los procesos, uno de ellos es la selección de proveedores de los materiales que se requieren para un proyecto, el $86,7 \%$ está de acuerdo en que la empresa realiza una selección de estos asegurándose que estás operen bajo el cumplimiento de las especificaciones técnicas y normas ambientales vigentes y solo el 13,3\% desconoce que esto se realice dentro de la organización. A su vez, el $60 \%$ está de acuerdo que dentro de la operación se minimiza el impacto ambiental negativo incrementando la eficiencia en la utilización de uso de materiales, energía y desarrollo de espacios, con el fin de eliminar desperdicios de tal manera que no se usen recursos por encima de lo necesario. 
La sostenibilidad como estrategia competitiva en empresas del sector construcción del departamento de Antioquia - Colombia

Tabla 5. Tendencias Indicador arquitectura y diseño sostenible

\begin{tabular}{|c|c|c|c|c|c|c|c|}
\hline $\begin{array}{l}\text { Indicador: } \\
\text { Arquitectura y } \\
\text { diseño } \\
\text { sostenible }\end{array}$ & $\begin{array}{l}\text { Se tiene en } \\
\text { cuenta el } \\
\text { sitio de } \\
\text { ejecución } \\
\text { del proyecto, } \\
\text { para evitar } \\
\text { trabajos en } \\
\text { áreas de } \\
\text { gran } \\
\text { contaminaci } \\
\text { ón } \\
\text { atmosférica }\end{array}$ & $\begin{array}{l}\text { Se } \\
\text { minimiza } \\
\text { el impacto } \\
\text { ambiental } \\
\text { negativo } \\
\text { al } \\
\text { increment } \\
\text { ar } \\
\text { eficiencia } \\
\text { en la } \\
\text { utilización } \\
\text { de uso de } \\
\text { materiales } \\
\text {, energía y } \\
\text { desarrollo } \\
\text { de } \\
\text { espacios }\end{array}$ & $\begin{array}{l}\text { Se reduce el } \\
\text { consumo de } \\
\text { energía para } \\
\text { calefacción, } \\
\text { iluminación y } \\
\text { otros } \\
\text { equipamiento } \\
\text { s, cubriendo } \\
\text { la demanda } \\
\text { con fuentes } \\
\text { de energía } \\
\text { renovable }\end{array}$ & $\begin{array}{l}\text { Los diseños } \\
\text { de los } \\
\text { proyectos se } \\
\text { realizan en } \\
\text { función de } \\
\text { los } \\
\text { condicionant } \\
\text { es climáticos } \\
\text { del lugar en } \\
\text { relación con } \\
\text { los } \\
\text { parámetros } \\
\text { arquitectónic } \\
\text { os }\end{array}$ & $\begin{array}{l}\text { Se } \\
\text { incorporan } \\
\text { mecanism } \\
\text { os para la } \\
\text { protección } \\
\text { de la } \\
\text { radiación } \\
\text { solar en } \\
\text { cualquier } \\
\text { época del } \\
\text { año }\end{array}$ & $\begin{array}{l}\text { Los } \\
\text { proyectos } \\
\text { se } \\
\text { acondicion } \\
\text { an y } \\
\text { preparan } \\
\text { para que } \\
\text { los } \\
\text { usuarios } \\
\text { realicen de } \\
\text { forma } \\
\text { selectiva la } \\
\text { entrega de } \\
\text { los } \\
\text { residuos } \\
\text { que } \\
\text { generan }\end{array}$ & $\begin{array}{l}\text { Se } \\
\text { seleccionan } \\
\text { proveedores } \\
\text { de materiales } \\
\text { que cumplan } \\
\text { con las } \\
\text { especificacion } \\
\text { es técnicas y } \\
\text { normas } \\
\text { ambientales } \\
\text { vigentes }\end{array}$ \\
\hline \begin{tabular}{l}
\multicolumn{2}{l}{ Tendencia } \\
Positiva (TA+ \\
DA)
\end{tabular} & $46,7 \%$ & $60,0 \%$ & $66,7 \%$ & $66,7 \%$ & $46,7 \%$ & $73,3 \%$ & $86,7 \%$ \\
\hline $\begin{array}{l}\text { Indiferencia } \\
\text { (NN) }\end{array}$ & $33,3 \%$ & $26,7 \%$ & $6,7 \%$ & $26,7 \%$ & $33,3 \%$ & $20,0 \%$ & $13,3 \%$ \\
\hline $\begin{array}{l}\text { Tendencia } \\
\text { Negativa (ED+ } \\
\text { TD) }\end{array}$ & $20,0 \%$ & $13,3 \%$ & $26,7 \%$ & $6,7 \%$ & $20,0 \%$ & $6,7 \%$ & $0,0 \%$ \\
\hline TOTAL & $100 \%$ & $100 \%$ & $100 \%$ & $100 \%$ & $100 \%$ & $100 \%$ & $100 \%$ \\
\hline
\end{tabular}

Fuente: elaboración propia

Por otro lado, los encuestados afirman que solo el $46,7 \%$ de las empresas tiene en cuenta el sitio de ejecución del proyecto para evitar trabajos en áreas de gran contaminación atmosférica, el $33,3 \%$ no tiene conocimiento y el $20 \%$ está en desacuerdo con que se realice. En la tabla $\mathrm{N}^{\circ} 5$ se encuentran los resultados correspondientes.

Con relación a esto, el $66,7 \%$ en base a $100 \%$ de los encuestados indican que en su empresa los diseños de los proyectos se realizan en función de los condicionantes climáticos del lugar en relación con los parámetros arquitectónicos, al considerar elementos como, por ejemplo: la temperatura del aire, la radiación solar, los vientos dominantes, entre otros. Esto implica que en las organizaciones se obligue a que todas las instalaciones tengan un plan que garantice el mantenimiento de los niveles de eficiencia energética y los parámetros de iluminación y solo el $73,3 \%$ de los encuestados reconoce que en su organización se lleve a cabo estos procesos dentro de la ejecución. También, el $66,7 \%$ indica que el consumo de energía para calefacción, iluminación y otros equipamientos se reduce con el fin de cubrir la demanda con fuentes de energía renovable, mientras que el $46,7 \%$ está de acuerdo en que se incorporan mecanismos para la protección de la radiación solar en cualquier época del año.

Una de las medidas utilizadas para minimizar los impactos negativos medio ambientales es el acondicionamiento y preparación de los proyectos para que los usuarios realicen de forma selectiva la entrega de los residuos que generan, donde el $73,3 \%$ de los encuestados confirman estar de acuerdo. Además de estas medidas, el $60 \%$ afirman que se 
diseñan redes separativas de evacuación de aguas residuales con el objetivo de reutilizarlas en otros usos (riego, incendios, inodoros). Los resultados están en sintonía con Wang et al., (2011), quienes señalan que, durante la ejecución del proyecto, se deben identificar actividades asociadas a especificaciones que pudieran afectar el diseño funcional de la obra, tales como: sistemas de agua, energía, ventilación, iluminación, entre otras consideraciones de tipo ambiental.

Hoy se ven grandes esfuerzos dentro de las empresas de construcción, reflejados en una buena implementación responsable en la arquitectura sostenible, cambiando la visión de los proyectos y mostrando un buen panorama frente a los cambios y adaptaciones que esto requiere. Sin embargo, es de gran importancia seguir trabajando en proyectos que sean cada vez más verdes y construir considerando una economía sostenible dentro de los mismos, donde los arquitectos cada vez planifican construcciones con materiales y diseños que ayuden a minimizar el uso de los recursos naturales y en la reutilización de la energía solar y el agua.

Seguidamente, se encuentra el indicador de ecoeficiencia, que es la manera de realizar un buen uso de los recursos con fines ecológicos en su máxima eficacia posible, ya sea en el uso del agua, el aire, el suelo, la energía, los desechos, la protección de la biodiversidad, entre otros.

A partir de la tabla $\mathrm{N}^{\circ} 6$, se puede observar una tendencia positiva en la que los encuestados reconocen en un $80 \%$ que la organización tiene una decisión de compra responsable frente a los productos que se utilizan dentro de los proyectos a diferencia de un $6,7 \%$ que expresa no estar de acuerdo, lo cual repercute en la minimización de la dependencia de los recursos naturales.

Tabla 6. Tendencias indicador de ecoeficiencia

\begin{tabular}{|c|c|c|c|c|}
\hline $\begin{array}{l}\text { Indicador: } \\
\text { Ecoeficiencia }\end{array}$ & $\begin{array}{l}\text { Se realiza un uso eficiente } \\
\text { del agua por medio de } \\
\text { políticas, instalación de } \\
\text { sistemas de ahorro, } \\
\text { reducción de consumo } \\
\text { desde los diferentes } \\
\text { labores y campañas para } \\
\text { promover el adecuado uso } \\
\text { por parte de los empleados }\end{array}$ & $\begin{array}{l}\text { Diseñan y desarrollan } \\
\text { lineamientos que la } \\
\text { empresa debe cumplir } \\
\text { dentro del proyecto } \\
\text { para } \\
\text { eficientemente } \\
\text { energíar }\end{array}$ & $\begin{array}{l}\text { Se tiene un uso eficiente } \\
\text { de transporte por medio de } \\
\text { la elección de vehículos } \\
\text { amigables con el medio } \\
\text { ambiente, el mantenimiento } \\
\text { de éstos y una buena } \\
\text { planeación de operación }\end{array}$ & $\begin{array}{l}\text { Se tiene una } \\
\text { decisión de compra } \\
\text { responsable frente } \\
\text { a los productos que } \\
\text { se utilizan dentro } \\
\text { de los proyectos }\end{array}$ \\
\hline $\begin{array}{l}\text { Tendencia } \\
\text { Positiva (TA+ DA) }\end{array}$ & $60,0 \%$ & $73,3 \%$ & $40,0 \%$ & $80,0 \%$ \\
\hline Indiferencia (NN) & $20,0 \%$ & $13,3 \%$ & $26,7 \%$ & $13,3 \%$ \\
\hline $\begin{array}{l}\text { Tendencia } \\
\text { Negativa (ED+ } \\
\text { TD) }\end{array}$ & $20,0 \%$ & $13,3 \%$ & $33,3 \%$ & $6,7 \%$ \\
\hline TOTAL & $100 \%$ & $100 \%$ & $100 \%$ & $100 \%$ \\
\hline
\end{tabular}

Fuente: elaboración propia 
La sostenibilidad como estrategia competitiva en empresas del sector construcción del departamento de Antioquia - Colombia

En relación con el uso eficiente de los recursos, el $73,3 \%$ de las organizaciones diseñan y desarrollan lineamientos que la empresa debe cumplir dentro del proyecto para usar eficientemente la energía y el $60 \%$ de estas realiza un uso eficiente del agua por medio de políticas, instalación de sistemas de ahorro, reducción de consumo desde los diferentes labores y campañas para promover el adecuado uso por parte de los empleados. Vale aclarar que el éxito de esto es poderlo integrar en las estrategias del proyecto desde sus etapas iniciales.

Sin embargo, el $40 \%$ indico el uso eficiente de transporte en el desarrollo de los proyectos en la organización por medio de la elección de vehículos amigables con el medio ambiente, el mantenimiento de éstos y una buena planeación de operación, frente a una tendencia negativa del $33,3 \%$ que expresan no estar de acuerdo con esto se implemente.

Bajo este orden de ideas, la investigación muestra datos que difieren del trabajo presentado por Enshassi (2018), donde se destacó la importancia de la incorporación de la gestión ambiental en los procesos de cada ciclo y etapa en el desarrollo de un proyecto, ya que allí se reveló los aspectos más importantes que, desde la práctica, consideran los ingenieros en cada fase de manera que todos los procesos desde el comienzo del diseño de la edificación hasta el final de su vida útil se realizaran de manera sostenible. En el departamento de Antioquia, los resultados arrojan aun discordancia en la aplicabilidad y falta de conocimiento en lo referente a la sostenibilidad en la construcción; y que, aun cuando algunas empresas están trabajando desde la arquitectura sostenible y la ecoeficiencia muchas carecen de la incorporación de estas prácticas. Sin embargo, el estudio valida la importancia de trabajar con los procesos enfocados en la sostenibilidad generando más seguridad a las personas y aumentando la conservación y el cuidado del medio ambiente.

En este sentido, el comportamiento de ambos indicadores, refleja una gran oportunidad de seguir trabajando en la contribución de la disminución del impacto negativo que generan las actividades humanas al medio ambiente. Es importante que las entidades gubernamentales sigan generando incentivos a las empresas y más a este sector que cumple un papel fundamental en este ámbito, donde puedan llevar a cabo políticas y estrategias en pro de la productividad y mejor uso de los recursos viéndose beneficiadas en la minimización de los costos de operación de los proyectos.

Finalmente, para evaluar las estrategias competitivas en las empresas del sector construcción, se midieron las dos ventajas que son diferenciación (de producto) y liderazgo en costos.

En primer lugar, en la tabla $\mathrm{N}^{0} 7$ se encuentran los resultados de la encuesta del indicador de liderazgo en costos, donde un $60 \%$ de los encuestados están de acuerdo en que la compañía reduce sus costos en los proyectos por medio de la automatización de procesos, lo que puede implicar una ventaja frente a sus competidores al tener costos inferiores.

De igual manera, esto se puede ver reflejado en que los encuestados confirman estar de acuerdo en un $60 \%$ en el que las empresas consideran la legislación del sector en el momento de diseñar procedimientos dirigidos a disminuir costos y en un $53,3 \%$ en que la aplicación de prácticas de construcción sostenible genera un efecto significativo en la disminución de los costos totales. 


\begin{tabular}{|c|c|c|c|c|c|}
\hline $\begin{array}{l}\text { Indicador: } \\
\text { Costos }\end{array}$ & $\begin{array}{l}\text { Se considera la } \\
\text { legislación del } \\
\text { sector en el } \\
\text { momento de diseñar } \\
\text { procedimientos } \\
\text { dirigidos a disminuir } \\
\text { costos }\end{array}$ & $\begin{array}{l}\text { La aplicación de } \\
\text { prácticas de } \\
\text { construcción } \\
\text { sostenible genera } \\
\text { un efecto } \\
\text { significativo en la } \\
\text { disminución de } \\
\text { costos totales }\end{array}$ & $\begin{array}{l}\text { La compañía } \\
\text { reduce sus costos } \\
\text { en los proyectos } \\
\text { por medio de la } \\
\text { automatización de } \\
\text { procesos }\end{array}$ & $\begin{array}{l}\text { La compañía } \\
\text { utiliza equipos } \\
\text { de última } \\
\text { generación para } \\
\text { ejecutar } \\
\text { proyectos en el } \\
\text { menor tiempo } \\
\text { posible }\end{array}$ & $\begin{array}{lr}\text { Se } & \text { tiene } \\
\text { implementado un } \\
\text { sistema } & \text { de } \\
\text { Gestión } & \text { de } \\
\text { Riesgos } & \\
\text { financieros } & \text { y } \\
\text { operativos } & \end{array}$ \\
\hline \begin{tabular}{ll}
\multicolumn{2}{l}{ Tendencia } \\
Positiva \\
DA)
\end{tabular} & $60,0 \%$ & $53,3 \%$ & $60,0 \%$ & $46,7 \%$ & $46,7 \%$ \\
\hline Indiferencia (NN) & $40,0 \%$ & $40,0 \%$ & $26,7 \%$ & $20,0 \%$ & $26,7 \%$ \\
\hline $\begin{array}{l}\text { Tendencia } \\
\text { Negativa (ED+ } \\
\text { TD) }\end{array}$ & $0,0 \%$ & $6,7 \%$ & $13,3 \%$ & $33,3 \%$ & $26,7 \%$ \\
\hline TOTAL & $100 \%$ & $100 \%$ & $100 \%$ & $100 \%$ & $100 \%$ \\
\hline
\end{tabular}

Fuente: elaboración propia

Sin embargo, se evidencia en un $26,7 \%$ que los encuestados están en desacuerdo en que las empresas tienen implementado un sistema de gestión de riesgos financieros y operativos, donde esto favorecería a la organización en la identificación de amenazas, obstáculos y oportunidades dentro de sus procesos. Una de estas reducciones de costos puede verse reflejado en la utilización de equipos de última generación para ejecutar proyectos en el menor tiempo posible, donde solo el $46,7 \%$ están de acuerdo en la implementación de estos dentro de la operación y un 33,3\% en desacuerdo.

Este indicador mostro una gran tendencia negativa e indiferencia en las respuestas de los encuestados, esto puede indicar que pocas empresas están trabajando con costos de producción más bajos y que pueden entrar a competir en el mercado con la ventaja competitiva en la disminución de precios frente a la competencia. Sin embargo, se debe tener en cuenta que para lograr esto se requiere de un control exhaustivo en todas las etapas del proyecto y que la gestión debe estar al alcance de todos.

Siguiendo con el orden de ideas, en la tabla №8 se evalúa la ventaja competitiva de diferenciación en producto siendo comparable con el de otra empresa, con base en esto el $80 \%$ de los encuestados en base al $100 \%$ está de acuerdo en que trabajar bajo los estándares de sostenibilidad genera una marcada diferenciación frente a la competencia.

Como se refleja en los resultados, el $46,7 \%$ de los encuestados reconocen también que se hacen visibles las acciones tomadas frente a los consumidores en temas de sostenibilidad en la organización y en un $66,7 \%$ en que la compañía se diferencia de su competencia por realizar proyectos con tecnologías innovadoras, donde se puede observar que se están realizando esfuerzos importantes para mejorar la oferta en comparación con sus competidores.

Para esto es indispensable evaluar que los proyectos ejecutados están alineados con la filosofía de gestión de la empresa preservando un enfoque de sostenibilidad, sin embargo, 
La sostenibilidad como estrategia competitiva en empresas del sector construcción del departamento de Antioquia - Colombia

solo el $46,7 \%$ está de acuerdo con que se realizan estos lineamientos dentro de su organización frente a un $26,7 \%$ que indica lo contrario. Aunque es necesario recalcar que esto comparado con un $53,3 \%$ de los encuestados que considera que la sostenibilidad es una variable determinante durante la negociación de algún proyecto.

Tabla 8. Tendencias indicador diferenciación

\begin{tabular}{|c|c|c|c|c|c|c|c|}
\hline $\begin{array}{l}\text { Indicador: } \\
\text { Diferenciació } \\
\text { n }\end{array}$ & $\begin{array}{l}\text { Trabajar } \\
\text { bajo los } \\
\text { estándares } \\
\text { de } \\
\text { sostenibilida } \\
\text { d genera } \\
\text { una marcada } \\
\text { diferenciació } \\
\text { n frente a la } \\
\text { competencia }\end{array}$ & $\begin{array}{l}\text { Los } \\
\text { proyectos } \\
\text { ejecutados } \\
\text { están } \\
\text { alineados } \\
\text { con la } \\
\text { filosofía de } \\
\text { gestión de la } \\
\text { empresa, } \\
\text { preservando } \\
\text { un enfoque } \\
\text { de } \\
\text { sostenibilida } \\
\text { d }\end{array}$ & $\begin{array}{l}\text { Las } \\
\text { alianzas } \\
\text { con sus } \\
\text { proveedore } \\
\text { s son } \\
\text { sólidas y en } \\
\text { conjunto } \\
\text { suman un } \\
\text { valor } \\
\text { diferencial } \\
\text { a la hora de } \\
\text { entregar a } \\
\text { tiempo las } \\
\text { obras }\end{array}$ & $\begin{array}{l}\text { La compañia } \\
\text { invierte en } \\
\text { innovación en } \\
\text { insumos de } \\
\text { uso más } \\
\text { frecuente a } \\
\text { partir de } \\
\text { nuevos } \\
\text { materiales } \\
\text { que se } \\
\text { encuentren } \\
\text { bajo los } \\
\text { estándares } \\
\text { reglamentado } \\
\text { s }\end{array}$ & $\begin{array}{l}\text { La } \\
\text { compañia } \\
\text { se } \\
\text { diferencia } \\
\text { de su } \\
\text { competenci } \\
\text { a por } \\
\text { realizar } \\
\text { proyectos } \\
\text { con } \\
\text { tecnologías } \\
\text { innovadora } \\
\text { s }\end{array}$ & $\begin{array}{l}\text { Utiliza } \\
\text { técnicas de } \\
\text { construcció } \\
n \\
\text { sostenible, } \\
\text { para } \\
\text { alcanzar } \\
\text { posiciones } \\
\text { de } \\
\text { liderazgo } \\
\text { en diseño y } \\
\text { ejecución } \\
\text { de } \\
\text { procesos }\end{array}$ & $\begin{array}{l}\text { La } \\
\text { sostenibilida } \\
\text { d es una } \\
\text { variable } \\
\text { determinant } \\
\text { e durante la } \\
\text { negociación } \\
\text { de algún } \\
\text { proyecto }\end{array}$ \\
\hline
\end{tabular}

\begin{tabular}{|c|c|c|c|c|c|c|c|}
\hline $\begin{array}{l}\text { Tendencia } \\
\text { Positiva (TA+ } \\
\text { DA) }\end{array}$ & $80,0 \%$ & $46,7 \%$ & $100,0 \%$ & $73,3 \%$ & $66,7 \%$ & $60,0 \%$ & $53,3 \%$ \\
\hline $\begin{array}{l}\text { Indiferencia } \\
\text { (NN) }\end{array}$ & $6,7 \%$ & $26,7 \%$ & $0,0 \%$ & $13,3 \%$ & $20,0 \%$ & $6,7 \%$ & $26,7 \%$ \\
\hline $\begin{array}{l}\text { Tendencia } \\
\text { Negativa (ED+ } \\
\text { TD) }\end{array}$ & $13,3 \%$ & $26,7 \%$ & $0,0 \%$ & $13,3 \%$ & $13,3 \%$ & $33,3 \%$ & $20,0 \%$ \\
\hline TOTAL & $100 \%$ & $100 \%$ & $100 \%$ & $100 \%$ & $100 \%$ & $100 \%$ & $100 \%$ \\
\hline
\end{tabular}

Fuente: elaboración propia

Por otro lado, el 73,3\% indica que la compañía invierte en innovación en insumos de uso más frecuente a partir de nuevos materiales que se encuentren bajo los estándares reglamentados y el $86,7 \%$ en que también se invierte en materiales de alta calidad porque su prioridad es ofrecer proyectos que sean bien valorados

Con relación a esto, se evidencia que el $60 \%$ de las compañías están utilizando técnicas de construcción sostenible, para alcanzar posiciones de liderazgo en diseño y ejecución de procesos, como por ejemplo en la confirmación en un $100 \%$ de que las organizaciones indican que las alianzas con sus proveedores son sólidas y en conjunto suman un valor diferencial a la hora de entregar a tiempo las obras.

El indicador de diferenciación, tomo una gran tendencia positiva en la evaluación de las empresas. Las empresas se están enfocando en ciertas características y gestiones sostenibles que hacen que los clientes las perciban como propuestas únicas a diferencia de su competencia, cabe destacar que las empresas cada vez están trabajando en acciones en pro de la sostenibilidad y que se ven reflejados en los esfuerzos que se realizan en las diferentes áreas para asumir con estos retos que las hacen sobresalir en un mercado tan saturado y rígido como lo es el sector de construcción. 
Los resultados evidencian frente a las estrategias competitivas que las empresas están trabajando en un entorno donde la diferenciación es la clave para destacar en este sector. Todavía queda un gran reto dentro de estas para poder trabajar con costos de producción más bajos, donde se hace posible con una gran gestión de implementación en todos los procesos de la organización, desde negociaciones con proveedores, adaptación de procesos, disminución y reutilización de recursos, entre otros. Las empresas deberán trabajar en las estrategias que construyan dicha ventaja competitiva sostenible en el tiempo.

\section{Conclusiones}

En relación al objetivo propuesto para esta investigación, el cual estuvo orientado en analizar la sostenibilidad como estrategia competitiva en las empresas del sector construcción del departamento de Antioquia, se concluye que las empresas del sector tienen una tendencia favorable en la implementación de la sostenibilidad en sus tres dimensiones, tanto en la gestión de sus proyectos como en las diferentes áreas funcionales, sin embargo, hoy se encuentra deficiencia en la implementación de estas como estrategia competitiva ya sea por costos o diferenciación.

La dimensión ambiental, fue uno de los indicadores con una mayor tendencia negativa en las respuestas de los encuestados. El sector construcción es uno de los más vigilados y regulados, pero aun así se ve gran debilidad en las prácticas de gestión ambiental donde se encontró que las organizaciones no se están enfocando en el mejoramiento de las estrategias en el uso eficiente de recursos y en la realización de prácticas sostenibles en los procesos para mitigar los impactos negativos al medio ambiente.

Para el indicador de sostenibilidad social, se encontró que las empresas en el sector tienen una mayor conciencia social en todos los ámbitos, desde el trato y contrataciones con los empleados, acuerdos con proveedores y hasta la planeación de estrategias y beneficios para las personas que gozarán de los proyectos que ejecuta cada organización. La dimensión económica obtuvo un mejor comportamiento, lo que quiere decir que las personas de este gremio resaltan que lo económico tiene un gran peso en todo su desarrollo industrial.

Con respecto a la aplicación de la sostenibilidad en las empresas del sector, se muestra que las organizaciones están realizando grandes esfuerzos reflejados en una buena implementación responsable en la arquitectura sostenible, cambiando la visión de los proyectos y mostrando un buen panorama frente a los cambios y adaptaciones que esto requiere. De igual forma, el comportamiento del indicador de ecoeficiencia, refleja una gran oportunidad de seguir trabajando en la contribución de la disminución del impacto negativo que generan las actividades humanas al medio ambiente en el uso eficiente de transporte en el desarrollo de los proyectos en la organización por medio de la elección de vehículos amigables y el mantenimiento regular de éstos.

Finalmente, con relación a las estrategias competitivas, se concluye que pocas empresas están trabajando con costos de producción más bajos y no se observa una distinción en donde puedan entrar a competir en el mercado con una estrategia asociada a la disminución de precios frente a la competencia. Sin embargo, se están enfocando en la estrategia competitiva de diferenciación en ciertas características que hacen que los clientes las perciban como propuestas únicas, estas están trabajando en acciones en pro de la sostenibilidad donde se ven reflejados los esfuerzos que se realizan en las diferentes áreas 
La sostenibilidad como estrategia competitiva en empresas del sector construcción del departamento de Antioquia - Colombia

para asumir retos que las hacen sobresalir en un mercado tan saturado y rígido como lo es el sector de construcción.

\section{Referencias Bibliográficas.}

Acevedo, Harlem, Vasquez, Alejandro, y Ramírez, Diego. (2012). Sostenibilidad: Actualidad y necesidad en el sector de la construccion en Colombia. Gestion y Ambiente, 15 (1), Colombia. (Pp. 105-118).

Álvaro, Ignacio. (2016). Desarrollo sostenible: la gran empresa de todas las empresas. El País. Extraído de: https://elpais.com/elpais/2016/12/26/planeta_futuro/1482755429_668116.html.

Argos. (2018). Tendencias de construcción sostenible en Colombia 2019. Extraído de: https://colombia.argos.co/tendencias-de-construccion-sostenible-en-colombia-2019/

BBVA. (2019). La importancia de la estrategia competitiva en la empresa. Extraído de: https://www.bbva.es/finanzas-vistazo/ef/empresas/estrategia-competitiva.html.

Bernyte, Sandra. (2018). Sustainability marketing communications based on consumer values and principles. Regional Formation and Development Studies, 26(3). Lituania. (Pp. 26-35). http://dx.doi.org/10.15181/rfds.v26i3.1807

Bradford, Henry. (2018). Sostenibilidad, un asunto de gestión empresarial. Semana Sostenible. Extraído de: https://sostenibilidad.semana.com/hablan-las marcas/articulo/sostenibilidad-un-asunto-de-gestionempresarial/41771.

CCCS. (2018). Programa LEED en Colombia. Extraído de: https://www.cccs.org.co/wp/capacitacion/talleres-de-preparacion-leed/.

Cruz, Orlando, y Álvarez, Lorena. (2014). Las ventajas competitivas sostenibles de las empresas de manufactura de calzado. Asociación Latinoamericana de Facultades y Escuelas de Contaduría y Administración. Panama.

Du Plessis, Chrisna. (2007), A strategic framework for sustainable construction in developing countries. Construction Management and Economics, Vol. 25, No. 1, Reino Unido. (Pp. 167-76). https://doi.org/10.1080/01446190600601313

Enshassi, Adnan. (2018), Environmental concerns for construction growth in Gaza Strip. Journal Building and Environment, Vol. 35, No. 3, China (Pp. 273-279). 10.1016/S0360-1323(99)00017-7.

Fernández, Catalina. (2014). Constructoras del Caribe le apuestan a la ejecución de obras sostenibles. La República. Extraído de: https://www.larepublica.co/economia/constructoras-del-caribe-le-apuestan-a-laejecucion-de-obras-sostenibles-2116041

Gallopín, Gilberto. (2003). Medio ambiente y desarrollo. Sostenibilidad y desarrollo sostenible: un enfoque sistémico. CEPAL. Chile.

Galvis, Gustavo. (2009). La sostenibilidad empresarial. Vanguardia. Colombia.

Garzón, Manuel e Ibarra, Alberto. (2014). Revisión Sobre la Sostenibildad Empresarial. Revista de Estudios Avanzados de Liderazgo, 1(July), España (Pp. 52-77)

Heincke, Mónica. (2005). La responsabilidad social empresarial: ¿Una herramienta para el desarrollo local sostenible en Colombia? Revista Opera, 5(5), Colombia, (Pp. 5574). 
Hoof, Bart y Herrera, Carlos. (2007). La evolución y el futuro de la producción más limpia en Colombia. Revista de Ingeniería. Universidad de Los Andes, 26, Colombia (Pp.101120). https://ojsrevistaing.uniandes.edu.co/ojs/index.php/revista/article/view/301/369

Hill, Richard y Bowen, Paul. (2010). Sustainable construction: principles and a framework for attainment. Revista Construction Management and Economics. España. Vol 15 (3). (Pp 223-239). https://doi.org/10.1080/014461997372971

LatinAmerican Post. (2019, February 14). ¿Por qué toda empresa debe ser sostenible? Extraído de: https://latinamericanpost.com/es/26394-por-que-toda-empresa-debe-sersostenible.

Leal, José. (2005). Ecoeficiencia: marco de análisis, indicadores y experiencias. CEPAL. Chile.

Li-Yin Shen, Jian Li Hao, Vivian Wing-Yan Tam y Hong Yao (2007). A checklist for assessing sustainability performance of construction projects. Journal of Civil Engineering and Management. Volumen 13 (4) China. (Pp 273-281). https://doi.org/10.3846/13923730.2007.9636447

Martínez, Jennifer. (2014). Consecuencias de las prácticas de sostenibilidad en el coste de capital y en la reputación corporativa. Revista de contabilidad. Vol. 17(2), España (Pp. 153-162). https://doi.org/10.1016/j.rcsar.2013.08.008

Meza, Alegría. (2007). La Responsabilidad Social Empresarial como Factor de Competitividad. Trabajo de grado para optar al título de Administradora de Empresas. Pontificia Universidad Javeriana. Colombia.

Ministerio del Ambiente Perú. (2009). Guía de Ecoeficiencia para Empresas. Ministerio Del Ambiente Perú. Extraído de: https://www.minam.gob.pe/calidadambiental/wpcontent/uploads/sites/22/2013/10/guia_de_ecoeficiencia_para_empresas.pdf

Porter, Michael. (2012). Ventaja Competitiva. Décima edición. Editorial McGraw-Hill. México.

Rodríguez, Carol. (2012). Sostenibilidad en las Empresas. Extraído de: https://www.eoi.es/blogs/carollirenerodriguez/2012/05/20/sostenibilidad-en-lasempresas/.

Rubio, Claudia. (2012). Gestión Estratégica Organizacional Aplicada a Las Pymes Constructoras En Colombia. Trabajo de Investigación para optar al título de Maestro en Creación y Dirección de empresas. Universidad EAN de España. España.

Villa, Fernando. (2009). Construcciones Verdes. Revista Alarife. Vol N 17, Colombia (Pp 3949).

Wang, Zanxin; Calderon, Margaret y Ying, Lu. (2011). Lifecycle assessment of the economic, environmental and energy performance of biodiesel in China. Journal Biomass and Bioenergy, Vol. 35, Reino Unido. (Pp. 2893-2902). https://doi.org/10.1016/j.biombioe.2011.03.031

Weaver, Alex; Pope, Jenifer; Saunders, Angus y Lochner, Paul. (2008), Contributing to sustainability as an environmental impact assessment practitioner. Impact Assessment and Project Appraisal, Vol. 26, No. 2, Reino Unido. (Pp. 91-98). https://doi.org/10.3152/146155108X316423 
La sostenibilidad como estrategia competitiva en empresas del sector construcción del departamento de Antioquia - Colombia

Yu, Chuck y Kim, Jeong. (2011). Building environmental assessment schemes for rating of IAQ in sustainable buildings. Indoor and Built Environment, Vol. 20, No. 1, China. (Pp. 5-15). https://doi.org/10.1177/1420326X10397780 